la revue La revue pour l'histoire du CNRS

POUR L'HISTOIRE DU CNRS

$7 \mid 2002$

La biologie | Menaces sur les sciences sociales vers

1980

\title{
Lamarck, genèse et enjeux du transformisme,
} 1770-1830

Pietro Corsi. CNRS ÉDITIONS, Paris, 2001

Hélène Blais

(2) OpenEdition

Journals

Édition électronique

URL : https://journals.openedition.org/histoire-cnrs/405

DOI : 10.4000/histoire-cnrs.405

ISSN : 1955-2408

Éditeur

CNRS Éditions

Édition imprimée

Date de publication : 5 novembre 2002

ISBN : 978-2-271-06067-9

ISSN : 1298-9800

Référence électronique

Hélène Blais, «Lamarck, genèse et enjeux du transformisme, 1770-1830 », La revue pour I'histoire du CNRS [En ligne], 7 | 2002, mis en ligne le 06 mars 2006, consulté le 21 septembre 2021. URL : http:// journals.openedition.org/histoire-cnrs/405; DOI : https://doi.org/10.4000/histoire-cnrs.405

Ce document a été généré automatiquement le 21 septembre 2021.

Comité pour l'histoire du CNRS 


\section{Lamarck, genèse et enjeux du transformisme, 1770-1830}

Pietro Corsi. CNRS ÉDITIONS, Paris, 2001

\section{Hélène Blais}

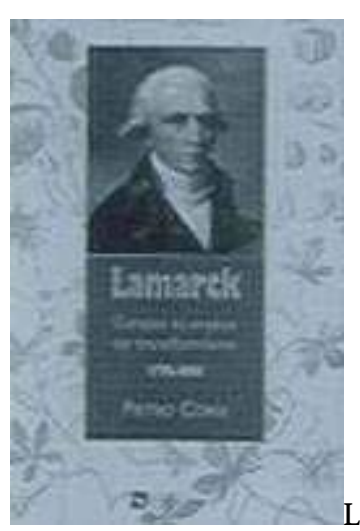

Le livre de Pietro Corsi, paru originellement en Italie en 1983, puis aux États-Unis en 1988, est enfin à la disposition du public francophone dans une édition mise à jour et enrichie, notamment grâce à l'utilisation d'une base de données initiée par l'auteur, qui recense l'ensemble des auditeurs de Lamarck (voir le site Internet http://www.lamarck.net). Le livre part d'un questionnement multiple sur le questionnement multiple sur le développement de la pensée de Lamarck, replacée dans le contexte des débats propres aux naturalistes entre les années 1770 et 1830 . L'originalité de la démarche réside dans l'exploitation systématique des principales publications scientifiques et culturelles de l'époque. Ainsi, l'examen de la logique interne de la pensée de Lamarck, souvent présentée comme isolée et détachée des recherches contemporaines, est étoffé ici d'une analyse de tous les éléments externes à cette pensée, permettant de la situer au mieux dans son contexte institutionnel et savant. Pietro Corsi remet ainsi en question l'historiographie traditionnelle qui voudrait laisser entendre que la pensée de Lamarck, dans son isolement, n'a eu aucune répercussion sur le débat naturaliste. Ce livre, fondé sur une analyse qui dissèque la chronologie, montre au contraire l'importance des discussions provoquées par les thèses de Lamarck. 
1 La démonstration tient en huit chapitres. Dans le premier, qui analyse le «projet de réforme de l'histoire naturelle ", Pietro Corsi revient sur la fin de l'ère Buffon en montrant que l'énorme succès éditorial de ce dernier bloque encore après sa mort en 1788 toute version autre de l'histoire naturelle. La période révolutionnaire bouleverse cependant le paysage en mettant en avant l'utilité pratique de la discipline, et en permettant l'émergence de jeunes savants motivés. L'époque est aussi celle de l'institutionnalisation de la discipline, avec la création du Muséum en 1793, de l'Institut en 1795. Ces instances de contrôle tendent à mettre un terme à la conception de l'histoire naturelle comme discipline littéraire, au profit de la reconnaissance d'une science académique. D'où l'apparition aussi de luttes et de controverses, que Pietro Corsi choisit de décrire en s'attachant à une chronologie très fine des débats naissants, notamment contre les théories de Buffon. Jusqu'en 1800, moment de la première formulation des hypothèses transformistes, des voix s'élèvent, comme celle de Daubenton ou de Vicq d'Azyr, qui témoignent de la pluralité des traditions scientifiques. C'est dans ce contexte de foisonnement d'idées que Pietro Corsi présente dans un deuxième temps, la formulation de la première synthèse transformiste. Précisant la chronologie des travaux de Lamarck, l'auteur pointe une rupture fondamentale en 1794, alors que le naturaliste inverse la chaîne des êtres vivants, accepte la génération spontanée et la transformation graduelle des formes de vie. Montrant sa volonté de convaincre, ses errements (notamment dans ses attaques contre la nouvelle chimie), analysant le jeu des réseaux d'influence dans le monde des naturalistes, l'historien met en lumière la complexité de la pensée de Lamarck, ici constamment étudiée en regard des sciences de l'époque (influence des travaux de Delamétherie, des débats contemporains sur l'histoire de la terre). Le troisième chapitre est consacré aux lois de la transformation et démontre que la formulation des hypothèses transformistes de Lamarck en 1800 s'inscrit dans un mouvement de pensée largement partagé par d'autres savants. Le débat sur les origines de la vie et notamment sur le questionnement en termes évolutifs de la succession des formes de la vie existe à la fin du XVIII ${ }^{e}$ siècle. C'est dans ce contexte qu'il faut donc relire le Discours de 1800 ou l'Hydrogéologie. Pietro Corsi souligne notamment l'importance du modèle dynamique et géologique dans la pensée transformiste de Lamarck, et montre que ce dernier, loin de penser dans sa bulle, écrit aussi en réaction à une certaine tendance de l'histoire naturelle qui se limiterait à la description des formes mortes. C'est dans ce cadre polémique qu'apparaît finalement la spécificité de la pensée de Lamarck. Le chapitre 4 est consacré principalement aux Recherches sur l'organisation des corps vivants (1802). L'œuvre est à nouveau interrogée dans son contexte, surtout pour ses silences par rapport aux débats de l'époque. L'historien des sciences montre ici la nécessité d'une attention soutenue aux démarches éditoriales, notamment dans la mention des travaux de ceux qui ne peuvent pas publier dans le cadre institutionnel de l'époque. Le chapitre 5 est consacré à la réception des Recherches. La rareté des réactions n'empêche pas de les considérer comme significatives. Mais elle demande une explication, que livre Pietro Corsi en rappelant les désaccords de Lamarck avec Cuvier, mais aussi la complexité de la pensée du premier, difficilement accessible. Le débat entre les deux hommes est éclairé d'un document nouveau, les notes d'un auditeur italien de Cuvier, qui confirment l'importance des dissensions institutionnelles entre les deux hommes. Très logiquement, le chapitre suivant traite des réactions de Lamarck aux critiques qui ont été formulées sur son travail, entre 1802 et 1809 (date de la parution de la Philosophie zoologique). Pietro Corsi interprète les faiblesses du débat en fonction de 
l'importance prise par les grands débats littéraires au détriment des disciplines de l'histoire naturelle. Il montre aussi les hésitations de Lamarck dans la confirmation de ses hypothèses, qui rendent toute analyse historique délicate pour cette période. La suivante, 1810-1830, est celle où sont étudiées les destinées du transformisme, les adhésions, souvent partielles, de certains savants, notamment de géologues, ou le dépassement de la théorie par un Bory de Saint Vincent. Le chapitre 8 est consacré au débat sur l'anatomie comparée entre Geoffroy Saint-Hilaire et Cuvier, débat dans lequel Pietro Corsi met en avant l'utilisation qui est alors faite du travail de Lamarck, utilisation avant tout stratégique. Au terme de ce développement subsistent des idées assez éloignées de la pensée de Lamarck, mais les adhésions au transformisme, même partielles, se multiplient à partir de 1825 , et témoignent de la constitution d'une culture pro-évolutionniste fondamentale dans la réception qui sera faite à De l'origine des espèces... de Darwin. Ainsi, l'ouvrage démontre brillamment l'importance des idées de Lamarck, tant pour elles-mêmes que pour leur rôle dans l'évolution de la pensée naturaliste. Il ouvre enfin des perspectives de recherche, puisque l'auteur évoque très rapidement en conclusion la diffusion du transformisme en Italie et en GrandeBretagne, ouvrant des horizons à l'étude entreprise, cette fois au-delà du cercle national.

INDEX

Mots-clés : Lamarck, darwinisme, évolution, transformisme, histoire naturelle

\section{AUTEUR}

HÉLÈNE BLAIS

Maître de conférences à l'université de Reims 